\title{
Heat flux distribution on a substrate in capacitively coupled radio-frequency discharges
}

\author{
K. Takaki, ${ }^{\text {a) }}$ D. Koseki, and T. Fujiwara \\ Department of Electrical and Electronic Engineering, Iwate University, Ueda 4-3-5, \\ Morioka 020-8551, Japan
}

(Received 18 July 2001; accepted for publication 2 January 2002)

\begin{abstract}
This letter investigates the heat flux distribution on a substrate in capacitively coupled radio-frequency argon glow discharges. The heat fluxes were determined from substrate temperature distributions measured by a high-sensitivity laser interferometer method with a $\mathrm{LiNbO}_{3}$ birefringent substrate. This method is based on monitoring the variation of refractive index with temperature. The distribution was obtained by sliding the $1-\mathrm{cm}$ long $\mathrm{LiNbO}_{3}$ substrate between two semicircular glass substrates placed on a disk electrode, $12 \mathrm{~cm}$ in diameter. The gap length was fixed at $2 \mathrm{~cm}$ and gas pressure was 100 mTorr. For the substrate placed on the powered electrode, the heat flux increases radically from the glow center to the radial electrode edge from 40 to $58 \mathrm{~mW} / \mathrm{cm}^{2}$. The heat flux toward the grounded electrode increases radically from 15 to $21 \mathrm{~mW} / \mathrm{cm}^{2}$. The total measured heat flux to the electrode is in good agreement with electrical power consumption determined from voltage and current measurements. The radial electron density profile in the discharge chamber was measured using a Langmuir probe. The results show that the maximum electron density observed at the electrode edge coincides with the maximum heat flux. (C) 2002 American Institute of Physics. [DOI: 10.1063/1.1456551]
\end{abstract}

It is well known that the power deposition in capacitively coupled radio-frequency discharges can be significantly nonuniform spatially. The effects of this nonuniform power deposition have been measured by optical emissions $s^{1,2}$ and predicted by models of glow discharges. ${ }^{3,4}$ However, the spatial distribution of the power deposition creates a nonuniform plasma density not only in the axial direction but also in the radial direction. ${ }^{5-8}$ Overzet and Hopkins have shown an increase of the electron density near the electrode edge in a Gaseous Electronics Conference reference reactor (GEC reactor). ${ }^{5}$ Boeuf and Pitchford showed similar M-shaped radial profiles of the electron density based on a twodimensional model and also showed ion flux profiles on the powered electrode. ${ }^{6}$ Therefore, the nonuniform ion flux causes nonuniform substrate temperature which affects chemical reactions.

The heat flux from the plasma to the substrate can be obtained using in situ surface temperature measurements. ${ }^{9}$ Though many methods have been used to measure substrate temperature, it is difficult to obtain the heat flux profile accurately on the substrate. ${ }^{10-13}$ For example, thermopiles, which are often used for local temperature measurements, disturb the electrical potential profile of the plasma. We have developed a method for noncontact measurement of the substrate temperature. ${ }^{14,15}$ This method is based on monitoring the variation on nature birefringence with temperature. The objectives of the experiment reported here are to characterize the heat flow from capacitively coupled radio-frequency plasma with respect to the heat flux distribution along the substrate surface and the power consumption.

The experimental investigations were performed using a symmetric parallel-plate plasma reactor, as shown in Fig. 1.

${ }^{a)}$ Electronic mail: takaki@iwate-u.ac.jp
The diameter of the upper and lower stainless electrodes is $12 \mathrm{~cm}$, the distance between the electrodes is $2 \mathrm{~cm}$, and the radial distance between the electrode edge and the chamber wall is $6 \mathrm{~cm}$. A stainless-steel mesh of $200 \mathrm{mesh} / \mathrm{in}$. was used as the ground shield. An insulator of $15 \mathrm{~cm}$ diam was inserted between the electrode and the shield. The argon gas flow through a mass flow controller is set to $250 \mathrm{cc} / \mathrm{min}$. The pressure was measured by an ionization vacuum gauge and controlled by a throttle valve in front of an oil diffusion pump. The lower electrode was cooled and kept at $20^{\circ} \mathrm{C}$ by recirculating water.

When we measured the heat flux toward the powered electrode, the lower electrode was powered by a $13.56 \mathrm{MHz}$ rf generator and the upper electrode was grounded. When we measured the heat flux toward the grounded electrode, we reversed the connection. The generator was connected to the line via a $\pi$-type matching network containing a coupling capacitor, which was introduced to obtain a direct-current bias between the powered electrode and the plasma.

The substrate temperature was monitored using the laser



FIG. 1. Schematic diagram of the experimental apparatus. The lower electrode was cooled by cycling $20^{\circ} \mathrm{C}$ water. 
interferometer as shown in Fig. 1. The optical measurement system consists of a He-Ne laser $(5 \mathrm{~mW}, 632.8 \mathrm{~nm}$ in wavelength), crossed polarizers, a photovoltaic detector, and a strip chart recorder. The laser beam of approximately $1 \mathrm{~mm}$ diam was directed to a $\mathrm{LiNbO}_{3}$ crystal substrate having the shape of a rectangular prism $(5 \times 2.5 \times 10 \mathrm{~mm}$ in width $\times$ height $\times$ length) after passing through the $45^{\circ}$ polarizer. The substrate was placed on the lower electrode. After passing through the substrate, a retardation occurred between the extraordinary ray and the ordinary ray. Using the crossed analyzer, the photovoltaic detector, and the chart recorder, the retardation was recorded as a function of time. The retardation of $2 \pi$ corresponds to a temperature change of $1.47{ }^{\circ} \mathrm{C}$ for the $1 \mathrm{~cm}$ length of the $\mathrm{LiNbO}_{3}$ substrate tip. ${ }^{16}$ By counting the number of fringes as a function of time, the data can be easily plotted as substrate temperature versus time. ${ }^{17}$ The experiments were repeated for various radial $\mathrm{LiNbO}_{3}$ tip positions.

The heat flux from plasma to substrate can be determined from a heat transport equation using measured substrate temperatures. The balance of the substrate between plasma gas heating and heat loss to the cooled electrode is given by ${ }^{11}$

$$
Q-h S \Delta T=c_{p} \rho d S \frac{d(\Delta T)}{d t},
$$

where $Q$ is the heat flux from plasma to the substrate, $h$ is the conductive heat transfer coefficient from the substrate bottom to the electrode, $\Delta T$ is the temperature difference between the substrate and electrode surface, $S$ is the area of the substrate, $t$ is time, $\rho$ is the mass density of the substrate, $c_{p}$ the specific heat, and $d$ the thickness of the substrate. In present work, $\rho, c_{p}, S$, and $d$ are $4.64 \mathrm{~g} \mathrm{~cm}^{-3}, 0.58$ $\mathrm{J} \mathrm{g}^{-1} \mathrm{~K}^{-1}, 0.5 \mathrm{~cm}^{2}$, and $0.25 \mathrm{~cm}$, respectively. Under the initial condition of $\Delta T=0$ at $t=0$, Eq. (1) becomes

$$
\Delta T=\frac{Q}{h S}\left[1-\exp \left(-\frac{t}{c_{p} \rho d S / h}\right)\right] .
$$

The conductive heat transfer coefficients $h$ can be estimated by fitting the temperature data with Eq. (2) and are obtained at $19 \mathrm{~W} \mathrm{~m}^{-2} \mathrm{~K}^{-1}$ for all $\mathrm{LiNbO}_{3}$ tip positions. When the substrate temperature is saturated, i.e., $d \Delta T / d t=0$, heat flux $Q$ can be estimated by using Eq. (1) with values of the saturated temperature rise $\Delta T s$ and $h$. The accuracy of this method was evaluated using a one-dimensional heat transport equation, and was within $4.9 \% .^{15}$ The distribution was obtained by sliding the $\mathrm{LiNbO}_{3}$ tip between two semicircular glass substrates placed on a disk electrode of $12 \mathrm{~cm}$ in diameter.

The potential of the powered electrode and discharge current were monitored by a $100 \mathrm{M} \Omega, 100 \mathrm{MHz}$ bandwidth 1:1000 voltage probe (Tektronix P6015A) and a current monitor (Pearson 2877), respectively. These signals were digitizing by a $1 \mathrm{GHz}$ sampling oscilloscope (SonyTektronix TDS 350) and transferred to a computer. Then, a correction for the propagation delay time of the cables was made to calculate the power consumption in the plasma. The plasma parameters, such as electron density, plasma potential, and electron temperature, were measured using a disktype Langmuir probe with a $2.9 \mathrm{~mm}$ diam.

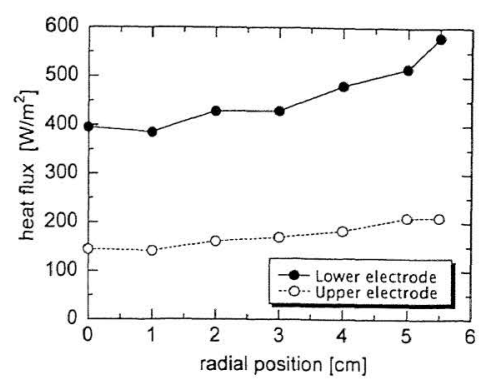

FIG. 2. Heat flux densities on the substrate measured using a $\mathrm{He}-\mathrm{Ne}$ laser interferometer with a $\mathrm{LiNbO}_{3}$ crystal in a 100 mTorr argon discharge at 335 $V_{\mathrm{pp}}$ discharge voltage. The heat flux toward the powered electrode ( ) and the grounded electrode $(O)$ were measured under the condition supplying the rf power to the lower and upper electrodes, respectively. The electrode center is at radial position zero and the electrode edge is at $6 \mathrm{~cm}$.

Figure 2 is a plot of the heat flux from the plasma to the substrate as a function of the radial position of the 1-cm-long $\mathrm{LiNbO}_{3}$ tip in an argon glow discharge at $100 \mathrm{~W}$ rf power and $100 \mathrm{~m}$ Torr gas pressure. The closed and open circles indicate the lower electrode was powered and grounded, respectively. The positions at 0 and $6 \mathrm{~cm}$ correspond to the electrode center and edge, respectively. For the powered electrode case, the heat flux increases from 40 to $58 \mathrm{~mW} / \mathrm{cm}^{2}$ with increasing radial position from the electrode center $(0$ $\mathrm{cm})$ to the electrode edge $(5.5 \mathrm{~cm})$. This value corresponds to approximately a $50 \%$ increase in heat flux. The heat fluxes toward the substrate on the grounded electrode are 14 and 21 $\mathrm{mW} / \mathrm{cm}^{2}$ at positions of 0 and $5.5 \mathrm{~cm}$, respectively. This value corresponds to a $50 \%$ increase with increasing radial position from the electrode center to the electrode edge. Note that the $50 \%$ increase occurs for both the powered electrode and the grounded electrode. The heat fluxes toward the grounded electrode, however, are smaller than the heat fluxes toward the powered electrode.

The total heat flux can be calculated using the following equation:

$$
\sum \Gamma_{h}=\sum_{k=1}^{n} \Gamma_{h k} S_{k},
$$

where $\Gamma_{h k}$ is the heat flux density at the $k$ th radial position, $n$ is the total number of points, and $S_{k}$ is the ring-shaped area shown as follows:

$$
S_{k}=\pi\left(r_{o k}^{2}-r_{i k}^{2}\right),
$$

where $r_{o k}$ and $r_{i k}$ are the $\mathrm{LiNbO}_{3}$ tip edge positions of the electrode edge side and the center side, respectively. The total heat fluxes obtained are approximately 5.6 and $2.1 \mathrm{~W}$ for the powered and the grounded electrode sides, respectively. These values are only $5.6 \%$ and $2.1 \%$ of the $100 \mathrm{~W}$ rf input power. The $2.1 \%$ total heat flux into the substrate on the grounded electrode is almost four times larger than the $0.5 \%$ total heat flux at the $4 \mathrm{~cm}$ gap length. ${ }^{17}$

Figure 3 is a plot of the electron density as a function of the radial position under the same discharge conditions as Fig. 2. The radial edge of the electrodes, the ground shields, and the chamber wall were at $6,7.5$, and $12 \mathrm{~cm}$, respectively. The center of the electrodes lies at $0 \mathrm{~cm}$. The electron density was measured three times at each position in order to reduce random error. All plots show the averaged values of these three measurement results. Figure 3 clearly shows that the 


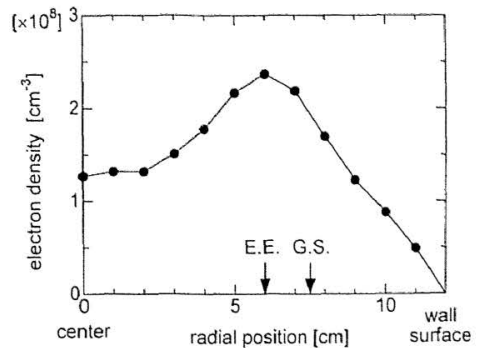

FIG. 3. Spatial distribution of the electron density measured using a Langmuir probe under the same conditions as Fig. 1. The electrode center is at radial position zero, the electrode edge (EE) at $6 \mathrm{~cm}$, the ground shield (GS) at $7.5 \mathrm{~cm}$, and the chamber wall at $12 \mathrm{~cm}$. The probe was placed at $1 \mathrm{~cm}$ from the upper electrode at the axial center of the gap.

electron density has the maximum point near the radial edge. The maximum value is approximately $80 \%$ larger than that of the electrode center under our experimental condition. Beyond the electrode edge, the electron density drops monotonically with position. The average diffusion flux for electrons and ions can be crudely estimated from the plasma density variation by using the argon-ion ambipolar diffusion coefficient

$$
D_{a}=D_{i}\left(1+\frac{T_{e}}{T_{i}}\right),
$$

where $D_{i}$ is the argon-ion diffusion coefficient, and $T_{e}$ and $T_{i}$ are the electron and ion temperatures, respectively. Using $T_{i}=300 \mathrm{~K}, T_{e}=2.7 \mathrm{eV}$, and $N D_{i}=2.07 \times 10^{18} \mathrm{~cm}^{-1} \mathrm{~s}^{-1}$ as the diffusion coefficient, ${ }^{18}$ the diffusion flux beyond the electrode edge is approximately $2.4 \times 10^{12} \mathrm{~cm}^{-2} \mathrm{~s}^{-1}$ and corresponds to the approximately $1.8 \times 10^{14} \mathrm{~s}^{-1}$ ion-electron pair loss rate. Assuming a monotonous decrease in both axial directions from the gap center, the diffusion flux to the electrodes would be approximately $8.1 \times 10^{12} \mathrm{~cm}^{-2} \mathrm{~s}^{-1}$ and corresponds to the approximately $1.8 \times 10^{15} \mathrm{~s}^{-1}$ ion-electron pair loss rate. Thus, the axial diffusion flux is ten times larger than the radial diffusion flux.

Figure 4 shows the wave forms of the potential close to the upper electrode and the current at the upper electrode under the same discharge condition as Fig. 2. The $100 \mathrm{~W}$ rf power was fed from the rf generator toward the upper electrode used as the powered electrode. The wave forms were corrected using 14.7 and $7.5 \mathrm{~ns}$ propagation times for the voltage and the current, respectively. Figure 4 shows the 335 $\mathrm{V}$ peak-to-peak (pp) voltage applied between the electrodes. The potential of the powered electrode is negatively biased, and these self-bias voltages correspond to $80 \%$ of the applied peak voltage. The plasma potential near the electrode edge was measured as $22 \mathrm{~V}$ with $15 \mathrm{~V}$ rf fluctuation by the probe measurements. The potential of the powered electrode is negatively biased to $-133 \mathrm{~V}$. Therefore, the time-averaged sheath voltage on the powered electrode can be estimated to $155 \mathrm{~V}$. The current wave form roughly agrees with the differentiated voltage with respect to time and with a multiplication factor of $0.15 \mathrm{nF}$.

The energy consumption per one cycle can be calculated by integrating instantaneous power (voltage times current), with respect to time for one cycle. The power consumption in the plasma can be determined by multiplying the energy

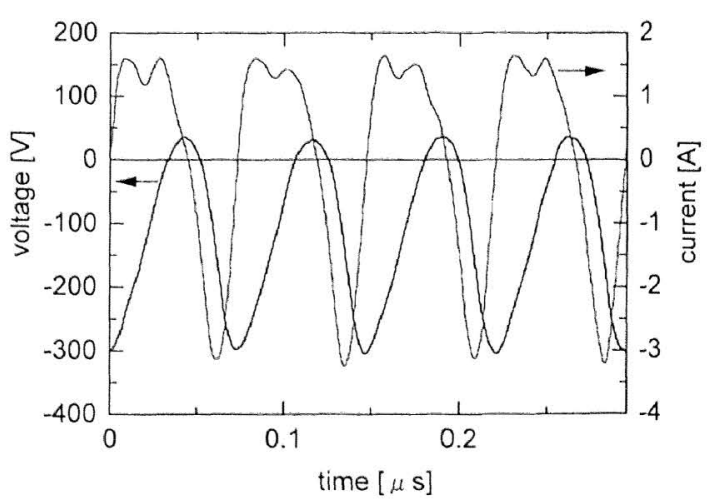

FIG. 4. Wave forms of the voltage on the powered electrode and the current at the powered electrode under the same conditions as Fig. 1.

by $13.56 \mathrm{MHz}$ rf frequency, and is thus obtained to $7 \mathrm{~W}$ when the $100 \mathrm{~W}$ rf power applied to the upper electrode through impedance matching circuits. The $2.1 \mathrm{~W}$ total heat flux toward the substrate on the lower grounded electrode corresponds to $30 \%$ of the energy consumed in the plasma. The voltage and current measurement system used in the present work is almost the same as that used at Wright Laboratory. ${ }^{19}$ The phase error in that measurement system using Tektronix P6015 and Pearson 2877 probes was reported to be less than $5^{\circ}$. However, it is necessary for more accurate determination of the consumed power that the measured voltages are corrected about the voltage drop between the measurement point and the electrode surface. The measured current also needs the correction about the current flow through a stray capacitance between the line and the ground. ${ }^{19}$

The authors would like to thank Professor N. Sato, and Dr. S. Mukaigawa of Iwate University, and Professor J. S. Chang of McMaster University for their valuable comments and discussions. The authors would also like to thank S. Kato and S. Kanno of Iwate University for their cooperation.

${ }^{1}$ F. Tochikubo, T. Kokubo, S. Kakuta, A. Suzuki, and T. Makabe, J. Phys. D 23, 1182 (1990).

${ }^{2} \mathrm{~N}$. Sato and Y. Shida, in Proceedings of the 11th International Conference on Gas Discharges and Their Applications, Tokyo, Japan, 11-15 September (1995) P-II-458.

${ }^{3}$ J. P. Boeuf, Phys. Rev. A 36, 2782 (1987).

${ }^{4}$ Y.-H. Oh, N.-H. Choi, and D.-I. Choi, J. Appl. Phys. 67, 3264 (1990).

${ }^{5}$ L. J. Overzet and M. B. Hopkins, Appl. Phys. Lett. 63, 2484 (1993).

${ }^{6}$ J. P. Boeuf and L. C. Pitchford, Phys. Rev. E 51, 1376 (1995).

${ }^{7}$ N. Sato and Y. Shida, Jpn. J. Appl. Phys., Part 1 36, 4794 (1997)

${ }^{8}$ M. Dalvie, M. Surendra, and G. S. Selwyn, Appl. Phys. Lett. 62, 3207 (1993).

${ }^{9}$ R. J. Visser, J. Vac. Sci. Technol. A 7, 189 (1989).

${ }^{10}$ R. Rochotzki and M. Friedrich, IEE Proc.-A: Sci., Meas. Technol. 139, 61 (1992).

${ }^{11}$ I. Hussla, K. Enke, H. Grunwald, G. Lorenz, and H. Stoll, J. Phys. D 20, 889 (1987).

${ }^{12}$ R. A. Bond, S. Dzioba, and H. M. Naguib, J. Vac. Sci. Technol. 18, 335 (1981).

${ }^{13}$ C. Foissac, P. Supiot, O. Dessaux, and P. Goudmand, Plasma Sources Sci. Technol. 8, 603 (1999).

${ }^{14}$ T. Fujiwara and H. Yamada, Rev. Sci. Instrum. 65, 267 (1994).

${ }^{15}$ K. Takaki, A. Takahashi, and T. Fujiwara, Jpn. J. Appl. Phys., Part 137, 3514 (1998)

${ }^{16}$ K. Takaki, K. Sayama, A. Takahashi, T. Fujiwara, M. Nagata, M. Ono, and M. A. Jani, Electr. Eng. Jpn. 127, 9 (1999).

${ }^{17}$ K. Takaki, A. Takahashi, and T. Fujiwara, J. Phys. D 33, 3060 (2000).

${ }^{18}$ D. P. Lymberopoulos and D. Economou, J. Appl. Phys. 73, 3668 (1993).

${ }^{19}$ P. J. Hargis, Jr. et al., Rev. Sci. Instrum. 65, 140 (1994). 